Ann. Génét. Sél. anim., 1975, 7 (1), 123-132.

\title{
ÉTUDE GÉNÉTIQUE DE L'ACTIVITÉ PRÉCOCE EN OPEN-FIELD DU JEUNE POUSSIN
}

\author{
J.-M. FAURE et J.-C. FOLMER \\ avec la collaboration technique de F. MorozEAU \\ Station Expérimentale d'Aviculture du Magneraud, I. N. R. A., \\ B.P. 52, 17700 Surgères (France)

\section{RÉSUMÉ}

Les poussins peuvent être séparés en deux types extrêmes quant à leur comportement en open-field : les actifs qui se déplacent et explorent beaucoup, qui émettent très fréquemment le piaulement, défèquent fréquemment et ont une latence courte et les inactifs présentant les caractéristiques inverses.

Nous avons sélectionné deux souches de poulets pour leur activité forte ou faible en openfield à l'âge de deux jours. Ce caractère est héritable et dès la première génération, les deux souches diffèrent significativement entre elles (nombre de tops, exploration, cris, déjections) et sont différentes de la souche témoin pour tous les caractères mesurés sauf les déjections à la troisième génération.

Les valeurs d'héritabilité relativement élevées (de 0,15 à 0,35 ) la précocité des mesures et leưr rapidité font que l'activité en open-field est un caractère très facilement sélectionnable.

\section{A. - INTRODUCTION}

Une mesure pour être utilisable pour des travaux de génétique doit être à la fois rapide, afin de pouvoir étudier le nombre maximum d'animaux et précise afin d’obtenir une héritabilité aussi élevée que possible ; de plus, il est souhaitable que la mesure soit prise le plus tôt possible afin de permettre une sélection précoce et de réduire les facteurs dus à l'apprentissage. Les sélections faites sur le comportement de la poule ont porté soit sur le comportement social (KOMAI, CraIG, WEARDEN, i959; Guhl, Craig, Mueliler, i960 ; Ortman, Craig, ig63; Craig, Ortman, GuHL, r965 ; Biswas, CraIG, I970) soit sur le comportement sexuel (SiEGEL, r959; SIEGEL, I965 ; SiEgel, HINKELMAN, I972).

L'inconvénient de ce type de sélection est que l'animal à mesurer doit réagir face à d'autres animaux donc à des conditions très variables, ce qui exige de nombreuses répétitions et nuit à la rapidité de la mesure. D'autre part chaque mesure est une valeur combinée entre deux génotypes. Au contraire, la mesure de la réaction 
de l'animal face au milieu (BROADHURST, I969 ; BOYER, MELIN, FERRÉ, I970 ; BOYER, MeL,IN, I974 ; Bourdens, I97I) se prête mieux à la sélection. L'open-field est de ce point de vue très pratique (BOYER et al., I970) et a donc choisi pour la rapidité des mesures qu'il permet.

\section{B. - MATÉRIEI ET MÉTHODE}

\section{1. - Appareil et méthode de mesure}

a) Description de l'open-field.

L'appareil (fig. I) est composé d'une cuve cylindrique de I $\mathrm{m}$ de diamètre et de $70 \mathrm{~cm}$ de haut. Les parois sont peintes en noir mat. Le fond est recouvert d'une couche de copeaux de bois. Le centre de la cuve (fig. I) est occupé par un prisme hexagonal dont les faces inclinées à $45^{\circ}$

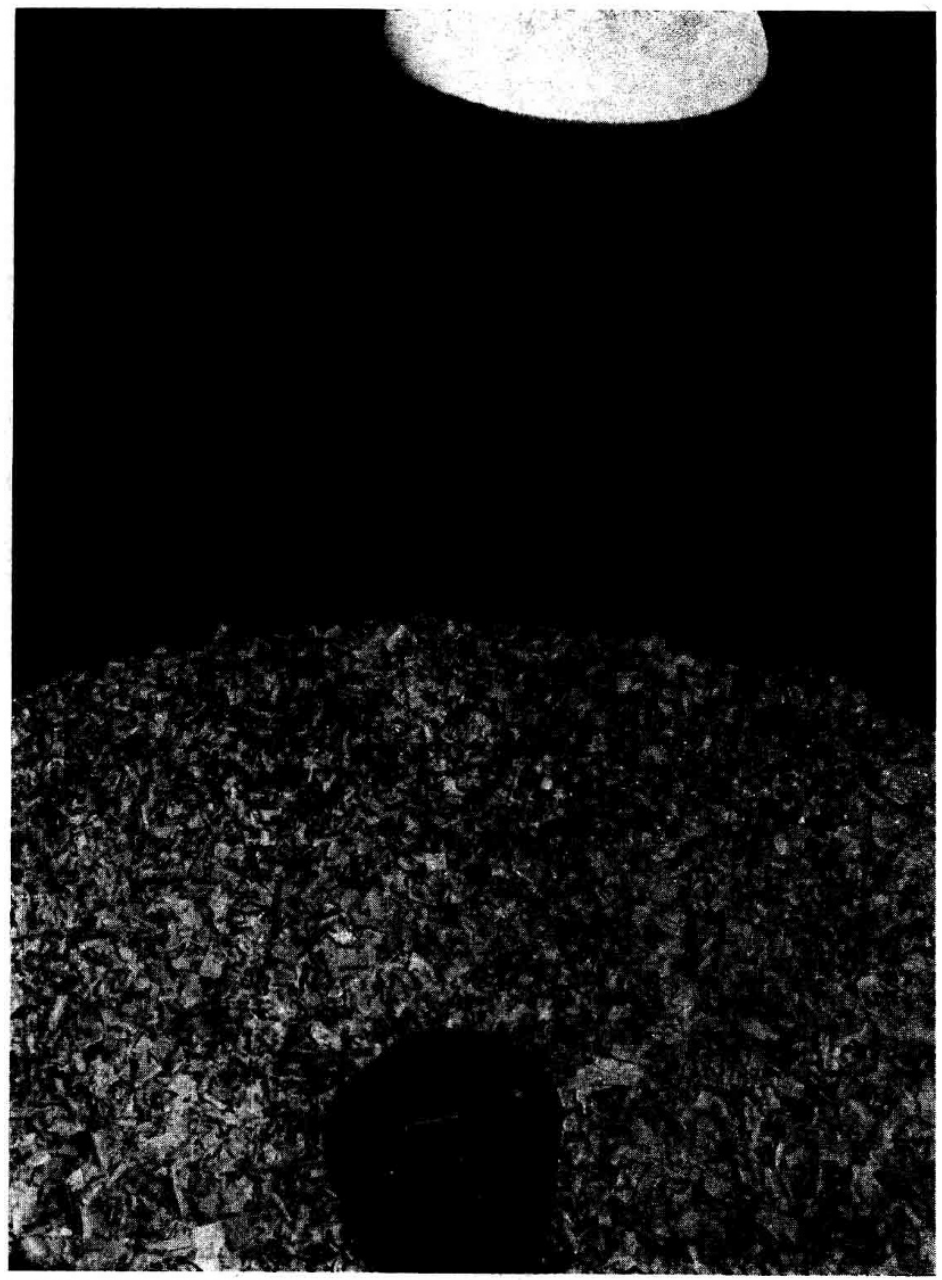

FIG. 1. - Photographie de l'open-field

On voit à la partie supérieure la lampe infrarouge, en bas au centre, le prisme réfléchissant à 6 faces 
renvoient la lumière produite par une lampe infrarouge de $250 \mathrm{~W}$ sur les 6 photo-transistors. Cette lampe sert simultanément à l'éclairage et au chauffage des poussins. L'ensemble du système d'automatisme est représenté par la figure 2.

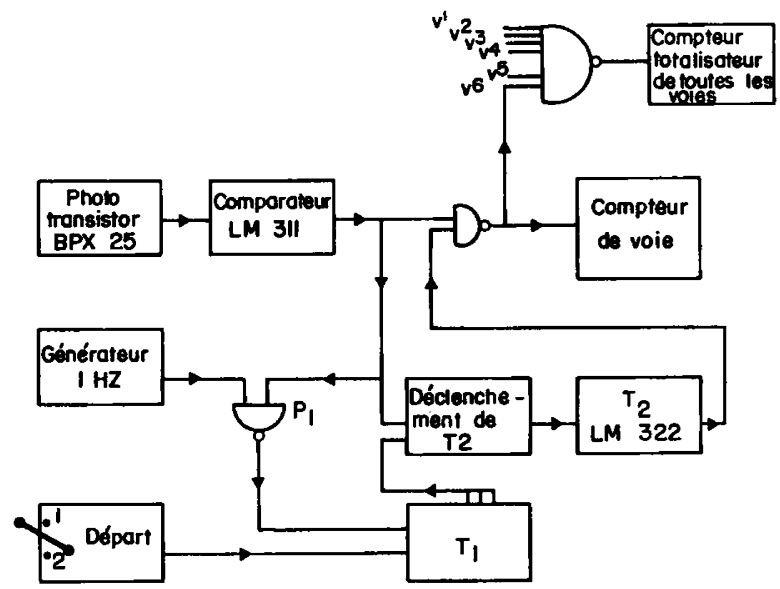

FIG. 2. - Schéma de principe du système d'automatisme et d'une voie de mesure

L'inverseur "Départ " dans sa position I met les compteurs à zéro, ainsi que les horloges $\mathrm{T}_{1}$ et $T_{2}$ du système d'automatisme. Lorsque cet inverseur est mis en position 2 , la porte $P_{1}$ se débloque, et laisse passer le signal $\mathrm{I}_{2}$ vers l'horloge digitale $\mathrm{T}_{1}$ comptabilisant ainsi la période de latence :

- si le poussin ne passe devant aucun photo-transistor, $T_{1}$ s'arrêtera sur une position prédéterminée (100 $\mathrm{s}$ dans le cas présent);

- si le poussin passe devant un photo-transistor, la tension collecteur de celui-ci diminue, ce qui entraîne un basculement du comparateur. A la sortie de ce comparateur, l'impulsion produite est dirigée vers trois directions :

- vers le compteur de la voie correspondant au photo-détecteur

- vers le compteur totalisateur de toutes les voies de l'appareil

- vers le déclenchement de $\mathrm{T}_{2}$

$T_{2}$ est un monostable à temporisation variable (Io nano-secondes à 3 heures), réglé dans le cas présent sur Ioo secondes.

L'impulsion issue du comparateur déclenche donc $T_{2}$ pour une durée de 100 secondes, quel que soit le nombre de passages devant chacun des photodétecteurs; et bloque l'horloge digitale $T_{1}$ qui affiche le temps de latence. Lorsque la période $T_{\mathbf{2}}$ est terminée les entrées compteurs se trouvent verrouillées le cycle de comptage est fini. L'ensemble électronique de cet appareil est réalisé en circuits intégrés digitaux TTL (transistor-transistor-logic).

\section{b) Méthode de mesure.}

Les animaux sont mis la veille du test dans une cage placée dans une pièce contiguë a la salle où se trouve l'open-field. Ils sont chauffés, nourris et abreuvés. L'animal à tester est placé entre 2 cellules et l'interrupteur de départ est mis en position 2. Si l'animal ne coupe pas un faisceau lumineux avant la fin des 100 secondes, il est noté latence $=100 \mathrm{~s}$, nombre de tops $=0$. Si l'animal coupe un faisceau lumineux avant la fin du $T_{1}$ les 6 compteurs et le totalisateur enregistrent le nombre de faisceaux interrompus (nombre de " tops") pendant roo secondes. En plus de la latence et du nombre de " tops " sont notés les caractères suivants :

- le nombre de cellules utilisées ;

- les cris suivant une notation allant de o à 2. Sont notés o les animaux ayant émis des piaulements pendant moins de 20 p. roo du temps, I entre 20 et 80 p. 100, 2 pour plus de 80 p. 100.

- la latence de la déjection (contrairement au mammifère, le nombre de déjections ne peut être compté car c'est un caractère tout ou rien).

- le nombre de sauts effectués contre les parois de l'open-field. 


\section{2. - Animaux et méthode d'élevage}

Les animaux proviennent d'une souche de type Cornish peu consanguine. La $\mathrm{F}_{0}$ a été composée de 400 poussins, provenant de 20 tot et 200 q pédigrés, éclos en un seul lot. Aux générations suivantes ro $\delta$ et 50 o par souche ont été utilisés pour la reproduction pédigrée et ont donné environ 400 poussins nés en deux lots d'éclosion séparés par I 5 jours. Jusqu'à l'âge de 8 semaines du lot I, les poussins sont élevés en séparant les souches et les lots, puis ensuite, en séparant les sexes et les souches; les animaux du même sexe et de la même souche étant regroupés quel que soit leur lot d'origine.

Il n'a pas été gardé de souche témoin sans sélection, nous avons cependant considéré comme témoin et mesuré comme tel à la $F_{8}$ la souche de départ qui n'est sélectionnée que pour ses caractéristiques de croissance.

$$
\text { 3. - Sélection }
$$

Le critère de sélection retenu est le produit du nombre de tops par le nombre de cellules utilisées : ce produit mesure mieux que le nombre de "tops " seul, l'activité de l'animal puisqu'un poussin peut effectuer un nombre de " tops " élevé sur une seule cellule et donc en se déplaçant très peu. La sélection est une sélection familiale tenant compte à la fois de la valeur de l'individu et de la valeur de ses frères et sœurs après transformation des valeurs brutes en probits afin de normaliser les distributions et d'annuler les effets lots (Cochez, PÉro, I954).

\section{C. - RÉSULTATS}

\section{I. - Description des comportements}

Typiquement, l'animal placé dans l'open-field est d'abord silencieux et immobile, puis il émet le gazouillis (twitter, pleasure call, ColiIAS (I952), CoLLIAS et Joos (I953), MONTEVECCHI et al. (I973)) ; ensuite le piaulement (peep, distress call, ColLIAS (I952), ColitiAs et Joos (r953); MONTEVECCHI et al. I973) avec très généralement un passage progressif entre les deux types de cris et ce n'est qu'après avoir émis les piaulements que l'animal commence à se déplacer. Cette succession a déjà été décrite par Guiron, 1959. La chaîne de réactions décrite peut se dérouler à des vitesses très variables suivant les individus et elle peut être interrompue à tous les stades par la fin du test. Une variante assez fréquemment observée est un départ instantané de l'animal au moment où il est posé dans l'open-field; il parcourt rapidement quelques dizaines de centimètres, s'arrête et éventuellement reprend la chaîne de réactions précédemment décrite.

\section{2. - Étude des répartitions des différentes mesures et de leurs liaisons à la $F_{0}$}

Dans tout ce qui suit, les sexes ne seront jamais séparés car dans la souche utilisée nous n'avons pu mettre en évidence aucune différence; ceci ne semble cependant pas être le cas pour toutes les souches.

L,es figures $3 a, b$ et $c$ illustrent respectivement la répartition du nombre de tops, de la latence et du nombre de cellules utilisées par les animaux de la $\mathrm{F}_{0}$.

On peut remarquer l'importance relative de la classe $o$ top (latence $=100 \mathrm{~s}$ ), qui comprend environ un tiers des animaux et l'asymétrie très marquée des répartitions du nombre de tops et de la latence. La relation entre nombre de tops et latence 
est représentée par la figure $3 d$. La corrélation entre ces deux mesures est de $-0,28$ $(P<I$ p. I 00o). La relation entre nombre de " top " et nombre de cellules utilisées est représentée par la figure 3 e. La corrélation est de 0,68 ( $\mathrm{P}<$ I p. I ooo), la relation est donc forte entre l'exploration et l'ambulation, et entre latence et ambulation. Par contre la corrélation entre nombre de cellules et latence est de $-0,08(P>5$ p. I0o). Toutes ces corrélations sont calculées sans tenir compte des poussins n'ayant pas démarré et qui ont tous une latence de roo s et un nombre de tops ou de cellules
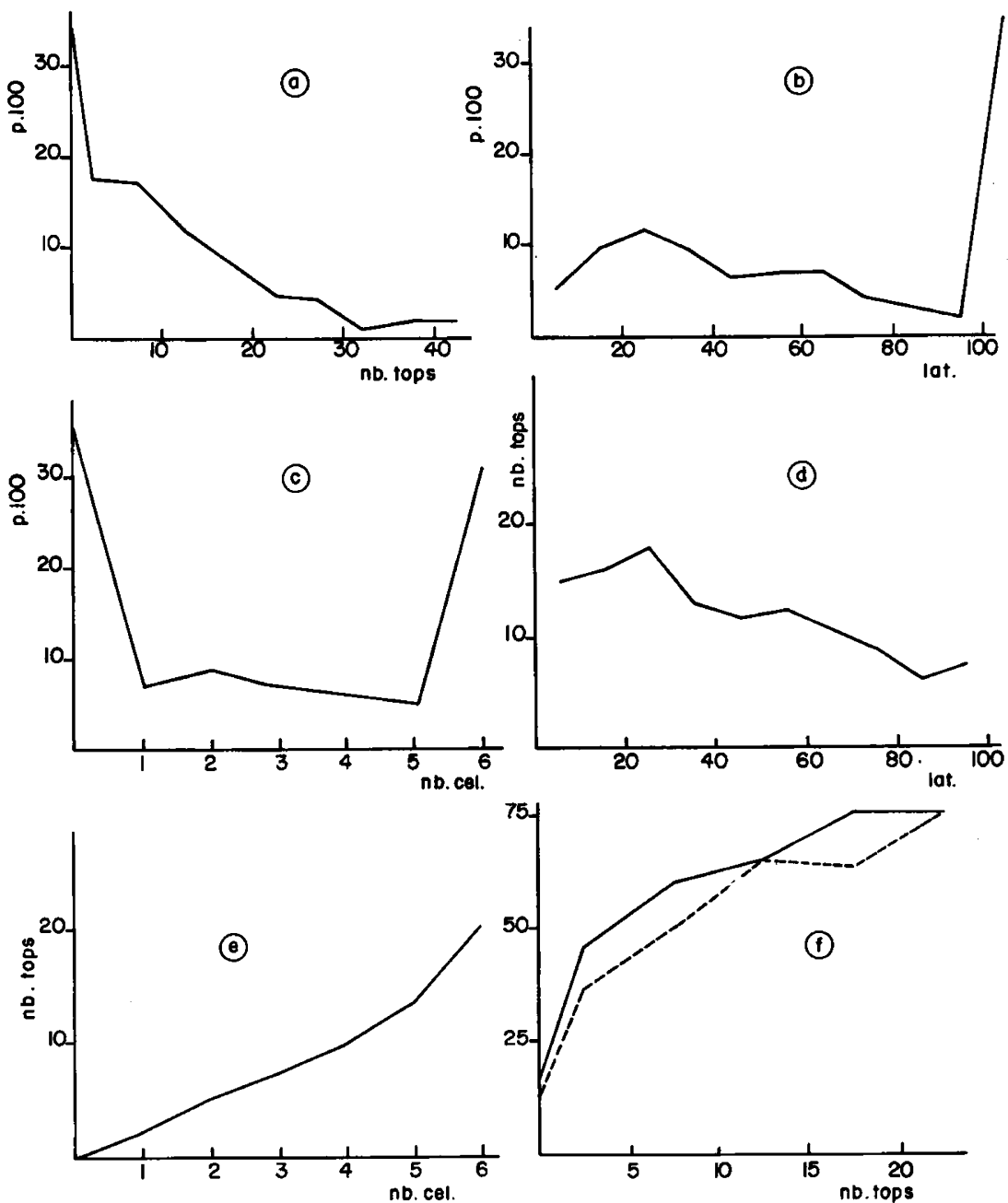

FIG. 3. - Distribution et relations des différents paramètres dans la génération de départ

$a$ : distribution du nombre de "tops"

$b$ : distribution des latences

$c$ : distribution du nombre de cellules utilisées

$d$ : relation entre latence et nombre de tops

$e$ : relation entre nombre de " tops et nombre de cellules

$f$ : en pointillés : pourcentage d'oiseaux déféquant pendant le test en fonction de l'activité.

En trait plein : pourcentage d'oiseaux émettant le cri de détresse pendant plus de $80 \mathrm{p}$. Ioo du temps 
égal à $\mathrm{o}$. Si on tient compte de tous les résultats on a alors la corrélation nombre de tops-latence $-0,72$; nombre de tops-nombre de cellules $0,8 \mathrm{I}$; nombre de celluleslatence $-0,49$.

Les relations entre nombre de " tops " et défécation d'une part, et nombre de " tops" et cris d'autre part, sont représentés par la figure $3 f$. On constate que les animaux actifs défèquent plus et émettent plus de piaulements que les inactifs.

Les actifs sont donc caractérisés par une exploration et une ambulation importantes, par une latence courte, des déjections fréquentes et une fréquence de piaulements élevée. Ces résultats sont comparables à ceux obtenus par BOYER et al. (I970, I974) sur le faisan et par ARCHER (I973) sur le poussin.

\section{3. - Évolution des souches}

La figure 4 représente en pourcentage cumulé la distribution du nombre de tops pour les 4 générations. On peut constater dans la souche I une évolution régulière portant aussi bien sur la proportion d'animaux notés o " top " que d'animaux très actifs. Par contre dans la souche $A$ la proportion de 0 " top " n'a pas varié de la $F_{1}$ à la $\mathrm{F}_{\mathrm{s}}$ mais on constate une augmentation régulière du nombre d'animaux très actifs

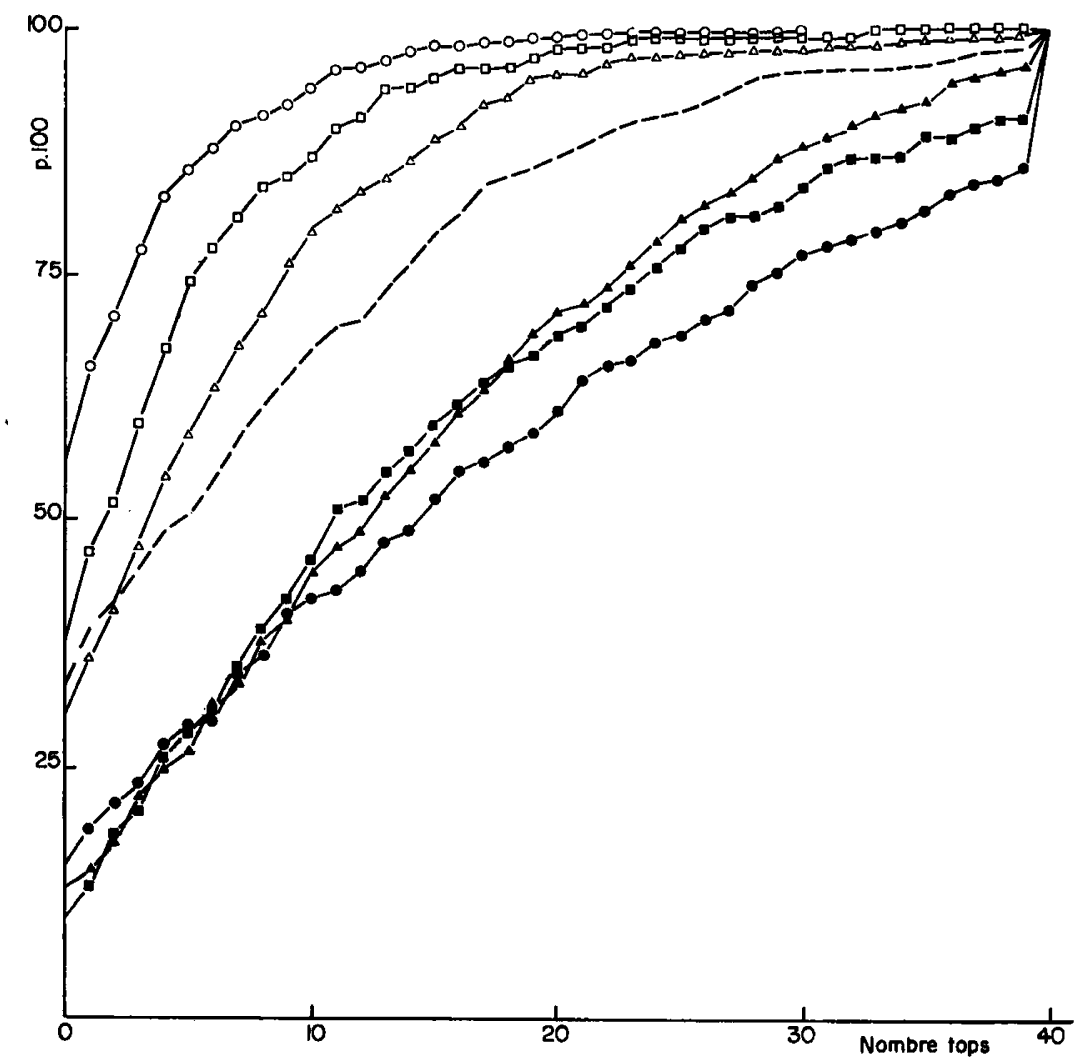

Fıg. 4. - Évolution du pourcentage cumule du nombre de "tops " au cours de la sélection

Tirets : population d'origine $\left(\mathrm{F}_{0}\right) \quad \Delta: \mathrm{I}^{\mathrm{re}}$ génération Figures vides : inactifs

Figures pleines : actifs 
(I2 p. Ioo des animaux font plus de 20 " tops " à la $F_{1}$, contre I6 p. Ioo à la $F_{2}$ et 23 p. Ioo à la $\mathrm{F}_{3}$ ) par contre le nombre de cellules utilisées (tabl. I) c'est-à-dire la proportion de la surface de 1'open-field explorée n'a pas varié de la $F_{1}$ à la $F_{3}$. Dans cette souche, ce n'est donc pas l'exploration qui a varié mais l'ambulation. On constate pour le caractère sélectionné (le produit du nombre de tops par le nombre de cellules) une divergence assez régulière dans les deux souches.

TABLEAU I

Evolution des souches

\begin{tabular}{|c|c|c|c|c|c|c|}
\hline \multirow{2}{*}{\multicolumn{2}{|c|}{ Caractère }} & \multicolumn{5}{|c|}{ Génération } \\
\hline & & $\mathrm{F}_{\mathbf{0}}$ & $\mathbf{F}_{1}$ & $\mathbf{F}_{2}$ & & $F_{3}$ \\
\hline $\begin{array}{c}\text { Nombre } \\
\text { d'animaux }\end{array}$ & $\begin{array}{c}\text { A } \\
\text { I }\end{array}$ & 402 & $\begin{array}{l}418 \\
367\end{array}$ & $\begin{array}{l}603 \\
492\end{array}$ & $\begin{array}{l}356 \\
315\end{array}$ & $100^{*}$ \\
\hline $\begin{array}{l}\text { Nombre } \\
\text { de tops }\end{array}$ & $\begin{array}{l}\text { A } \\
\text { I }\end{array}$ & 9,48 & $\begin{array}{r}15,73 \\
7,07\end{array}$ & $\begin{array}{r}15,78 \\
4,11\end{array}$ & $\begin{array}{r}21,50 \\
2,28\end{array}$ & 11,09 \\
\hline $\begin{array}{c}\text { Nombre } \\
\text { de cellules }\end{array}$ & $\begin{array}{l}\text { A } \\
\text { I }\end{array}$ & 2,80 & $\begin{array}{l}4,11 \\
2,39\end{array}$ & $\begin{array}{l}4,13 \\
1,72\end{array}$ & $\begin{array}{l}3,97 \\
1,04\end{array}$ & 3,17 \\
\hline $\begin{array}{c}\text { Produit } \\
\text { nombre tops } \\
\text { nombre de cellules }\end{array}$ & $\begin{array}{l}\text { A } \\
\text { I }\end{array}$ & 45,58 & $\begin{array}{l}81,92 \\
26,24\end{array}$ & $\begin{array}{l}86,90 \\
14,55\end{array}$ & $\begin{array}{r}104,33 \\
7,62\end{array}$ & 55,66 \\
\hline Latence & $\begin{array}{l}\text { A } \\
\text { I }\end{array}$ & 50,25 & $\begin{array}{l}37,71 \\
41,54\end{array}$ & $\begin{array}{l}27,82 \\
52,90\end{array}$ & $\begin{array}{l}38,82 \\
68,92\end{array}$ & 51,61 \\
\hline $\begin{array}{l}\text { Pourcentage } \\
\text { de déjections }\end{array}$ & $\begin{array}{c}\text { A } \\
\text { I }\end{array}$ & 42 & $\begin{array}{l}44 \\
30\end{array}$ & $\begin{array}{l}48 \\
30\end{array}$ & $\begin{array}{l}45 \\
28\end{array}$ & 36 \\
\hline $\begin{array}{l}\text { Pourcentage } \\
\text { de notés } 2 \\
\text { pour les cris }\end{array}$ & $\begin{array}{c}\text { A } \\
\text { I }\end{array}$ & 47 & $\begin{array}{l}69 \\
48\end{array}$ & $\begin{array}{l}72 \\
40\end{array}$ & $\begin{array}{l}79 \\
45\end{array}$ & 63 \\
\hline
\end{tabular}

* Les valeurs données dans cette colonne sont celles qui ont été obtenues sur des animaux de la souche de départ mesurés en même temps que le animaux de la $F_{3}$.

On constate aussi que les autres caractères mesurés et qui avaient été trouvés en corrélation avec l'activité ont tous divergé au cours de la sélection (toutes les mesures sont significativement différentes entre $A$ et $I$ dès la $F_{1}$ au seuil I $p$. Ioo, sauf pour la latence pour laquelle les différences n'atteignent le seuil I p. Ioo qu'à partir de la $F_{2}$ ) avec cependant des tendances au plateau pour la latence et le pourcentage de déjections chez les " actifs" et pour les cris chez les "inactifs ". D'autre part, à la $\mathrm{F}_{\mathbf{3}}$ toutes les mesures sont différentes (seuil I p. roo) de celles des témoins dans les deux souches, sauf pour le pourcentage de déjections. 
Le tableau 2 donne, les valeurs d'héritabilité calculées et réalisées pour les caractères continus (nombre de " tops ", latence et produit) et les valeurs calculées pour les deux caractères tout ou rien (les cris et les déjections).

TABLEAU 2

Valeur des héritabilités père-mère calculées et réalisées

\begin{tabular}{|c|c|c|c|c|c|c|c|}
\hline Caractère & $\begin{array}{c}\text { Type } \\
\text { d'hérita- } \\
\text { bilité }\end{array}$ & Souche & $F_{0}$ & $F_{1}$ & $F_{2}$ & $\mathrm{~F}_{3}$ & $\begin{array}{l}\text { Valeurs } \\
\text { moyennes }\end{array}$ \\
\hline \multirow{2}{*}{$\begin{array}{l}\text { Nombre } \\
\text { de tops }\end{array}$} & calculée & $\begin{array}{l}\text { A } \\
\text { I }\end{array}$ & 0,32 & $\begin{array}{l}0,13 \\
0,05\end{array}$ & $\begin{array}{l}0,20 \\
0,03\end{array}$ & $\begin{array}{l}0,00 \\
0,27\end{array}$ & 0,14 \\
\hline & réalisée & $\begin{array}{l}\text { A } \\
\text { I }\end{array}$ & & $\begin{array}{l}0,55 \\
0,25\end{array}$ & $\begin{array}{l}0,00 \\
0,49\end{array}$ & $\begin{array}{l}0,44 \\
0,56\end{array}$ & 0,38 \\
\hline \multirow{2}{*}{ Latence } & calculée & $\begin{array}{l}\text { A } \\
\text { I }\end{array}$ & 0,30 & $\begin{array}{l}0,35 \\
0,10\end{array}$ & $\begin{array}{l}0,08 \\
0,04\end{array}$ & $\begin{array}{l}0,08 \\
0,17\end{array}$ & 0,16 \\
\hline & réalisée & $\begin{array}{l}\text { A } \\
\text { I }\end{array}$ & & $\begin{array}{l}0,38 \\
0,17\end{array}$ & $\begin{array}{l}0,52 \\
0,30\end{array}$ & $\begin{array}{r}-1,65 \\
0,50\end{array}$ & $\begin{array}{c}0,04 \\
(0,37)^{*}\end{array}$ \\
\hline \multirow{2}{*}{$\begin{array}{c}\text { Produit } \\
\text { nombre tops } \\
\text { nombre cell. }\end{array}$} & calculée & $\begin{array}{l}\text { A } \\
\text { I }\end{array}$ & 0,40 & $\begin{array}{l}0,15 \\
0,07\end{array}$ & $\begin{array}{l}0,22 \\
0,01\end{array}$ & $\begin{array}{l}0,00 \\
0,28\end{array}$ & 0,16 \\
\hline & réalisée & $\begin{array}{l}\text { A } \\
\text { I }\end{array}$ & & $\begin{array}{l}0,49 \\
0,42\end{array}$ & $\begin{array}{l}0,04 \\
0,48\end{array}$ & $\begin{array}{l}0,21 \\
0,52\end{array}$ & 0,36 \\
\hline Cris & calculée & $\begin{array}{c}\text { A } \\
\text { I }\end{array}$ & 0,22 & $\begin{array}{l}0,36 \\
0,20\end{array}$ & $\begin{array}{l}0,07 \\
0,29\end{array}$ & $\begin{array}{l}0,24 \\
0,20\end{array}$ & 0,23 \\
\hline Déject. & calculée & $\begin{array}{l}\text { A } \\
\text { I }\end{array}$ & 0,05 & $\begin{array}{l}0,02 \\
0,11\end{array}$ & $\begin{array}{l}0,06 \\
0,12\end{array}$ & $\begin{array}{l}0,21 \\
0,17\end{array}$ & 0,11 \\
\hline
\end{tabular}

* : Valeur calculée sans tenir compte de la valeur aberrante obtenue pour les actifs $\mathrm{F}_{3}$.

Pour les trois caractères continus, l'héritabilité a été calculée après transformation des valeurs en probits afin de normaliser les distributions. La dernière colonne du tableau donne les valeurs moyennes de l'héritabilité. On peut constater que pour les trois caractères continus, les valeurs moyennes de l'héritabilité calculée sont de l'ordre de 0,15 ; alors que les valeurs moyennes de l'héritabilité réalisée sont de l'ordre de 0,35 . Ces divergences peuvent s'expliquer par la forme très asymétrique des distributions (fig. $3 a$ et $b$ ), le calcul de l'héritabilité étant prévu avec une répartition normale des valeurs. Pour les deux caractères tout ou rien les valeurs moyennes sont faibles surtout pour les déjections, ce qui pourrait expliquer que pour ce caractère, les deux souches ne diffèrent pas significativement du témoin. 


\title{
D. - DISCUSSION
}

Nous avons pu sur les caractères de comportement en "open-field ", séparer les jeunes poussins en deux types extrêmes : les actifs explorant la plus grande partie de l'open-field, se déplaçant beaucoup, à latence courte, qui émettent beaucoup de piaulements et défèquent fréquemment et les inactifs présentant les caractères inverses. Ces caractères sont pour la plupart héritables et une sélection divergente portant sur l'activité en " open-field" a permis de faire diverger les deux souches sur tous les caractères mesurés.

$\mathrm{Si}$ on compare ces résultats à ceux obtenus chez le Rat par BROADHURST (1960), on constate que les animaux les plus actifs sont ceux qui défèquent le plus, alors que chez le Rat, on a la relation inverse. Ceci montre que les critères permettant de mesurer l'émotivité ne sont pas les mêmes dans les deux espèces. Les valeurs d'héritabilité trouvées sont de l'ordre de 0,20 à 0,40 , c'est-à-dire très comparables à ce qui a été trouvé chez la même espèce pour l'aptitude à la dominance (Komar et al., I959), pour l'activité sexuelle des coqs (SIEGEI, I972) ; chez le Faisan et la Poule pour 1'activité en " open-field" (BOyER et al., I970, I973, I974; BOURDENS, I97I) ou chez la drosophile pour la vitesse de copulation (MANNING, I96I).

Ceci confirme que les caractères de comportement présentent des héritabilités suffisantes pour être sélectionnés dans des souches utilisées pour la production. Le principal obstacle à la sélection des souches commerciales est la lenteur des mesures de comportement. Nos résultats montrent que bien que les mesures soient prises pendant un temps très court, la précision est suffisante pour obtenir des valeurs d'héritabilité utilisables. Il faudra cependant préciser les liaisons existant entre 1a mesure précoce en open-field et des caractères intéressant l'aviculteur et parmi ceux-ci l'émotivité avant que cette méthode puisse être utilisée pratiquement.

Reçu pour publication en mars 1975.

\section{SUMMARY}

\author{
GENETIC STUDY OF EARLY ACTIVITY \\ IN OPEN-FIELD OF THE YOUNG CHICKEN
}

\footnotetext{
Chicks can be divided into two extreme types as for their behaviour in open-field : active ones which move and explore very much, which very often utter peepings, often defecate and have a short latency, and inactive ones presenting opposite characteristics.

We selected two strains of chickens for their high or low activity in open-field, at two days of age. This character is heritable and from the first generation, the two strains significantly differ from one another (number of tops, exploration, calls, dejections) and are different from the control strain in all measured characters except dejections at third generation.

The result of rather high values of heritability (o.15-0.35), earliness of measures and their rapidity is that activity in open-field is a very easy character to select.
} 


\section{RÉFÉRENCES BIBLIOGRAPHIQUES}

ARcher J., 1973. The influence of testosterone on chick behavior in novel environments. Behav. Biol., 8, 93-ro8.

BISwas D. K., CRAIG J. V., I97o. Genotype environment interractions in chickens selected for high and low social dominance." Poultry Sci., 49, 681-692.

Bourdens P., r97r. Le Faisan de repeuplement. Une approche par la génétique du comportement. Essais de sélection par des tests précoces. Mémoire de fin d'études, E. S. A., Purpan-Toulouse.

Boyer J.-P., Melin J.-M., FerRt R., I97o. Différences génétiques de comportement exploratoire expérimental chez le poussin. Premiers résultats. XIV World's Poult. Cong., Madrid, 2, 2 1-25.

Boyer J.-P., Melin J.-M., Bourdens P., 1973. Activity test on young pheasants. Ann. Génét. Sêl. Anim., 5, 4I7-4I8.

Boyer J.-P., Melin J.-M., I974. Une expérience d'élevage et de sélection du Faisan de repeuplement. Colloque I. T. A. V. I., Gibier, Périgueux, 37-43.

Broadhurst P. L., 1969. Psychogenetics of emotionality in the rat. Ann. N. Y. Acad. Sci., 159, 806-824.

Cochez L. P., Pero L., r954. Transformation des mesures brutes en écarts réduits pour atténuer l'incidence des variations de milieu. $X$ World's Poult. Cong., Édinburgh, I6-I9.

Collias N. E., I952. The development of social behavior in birds. $A u k, 69$, I27-I59.

Collias N. E., Joos M., I953. The spectrographic analysis of signals of the domestic fowl. Behavior, 5, r76-r 88.

Cook W. T., Siegel P. B., Hinkelmann K., 1972. Genetic analysis of male mating behavior in chickens. II. Crosses among selected and control lines. Behavior genetics, 2, 289-300.

Craig J. V., Ortman L. L., Guhl A. M., 1965. Genetic selection for social dominance ability in chickens. Anim. Behav., 18, II4-I3I.

Guiton P., 1959. Socialization and imprinting in Brown Leghorn chicks. Anim. Behav., 7, 26-34.

Guhl A. M., Craig J. V., Mueller C. D., I96o. Selective breeding for aggressiveness in chickens. Poultry Sci., 39, 970-980.

KomaI T., CRAIG J. V., WEARDEN S., I959. Heritability and repeatability of social aggressiveness in the domestic chicken. Poultry Sci., 38, 356-359.

Manning A., I96r. The effects of artificial selection for mating speed in Drosophila melanogaster. Anim. Behav., 9, 82-92.

Montevecchi W. A., Gallup G. G., Dunlap W. P., 1973. The peep vocalization in group reared chicks (Gallus domesticus) : its relation to fear. Anim. Behav., 21, 1 I6-123.

Ortman L. L., Craig J. V., I963. The development of aggressive behavior in selected strains of chickens. Poultry Sci., 42, 1296.

SIEGEL P. B., x959. Evidence of a genetic basis for aggressiveness and sex drive in the white plymouth rock cock. Poultry Sci., 38, II5-II8.

SIEGEL P. B., 1965. Genetics of behavior : selection for mating ability in chickens. Genetics, 52, I269I277.

SIEGEL P. B., 1972. Genetic analysis of male mating behavior in chickens (Gallus domesticus). I. Artificial selection. Anim. Behav., 20, 564-570. 